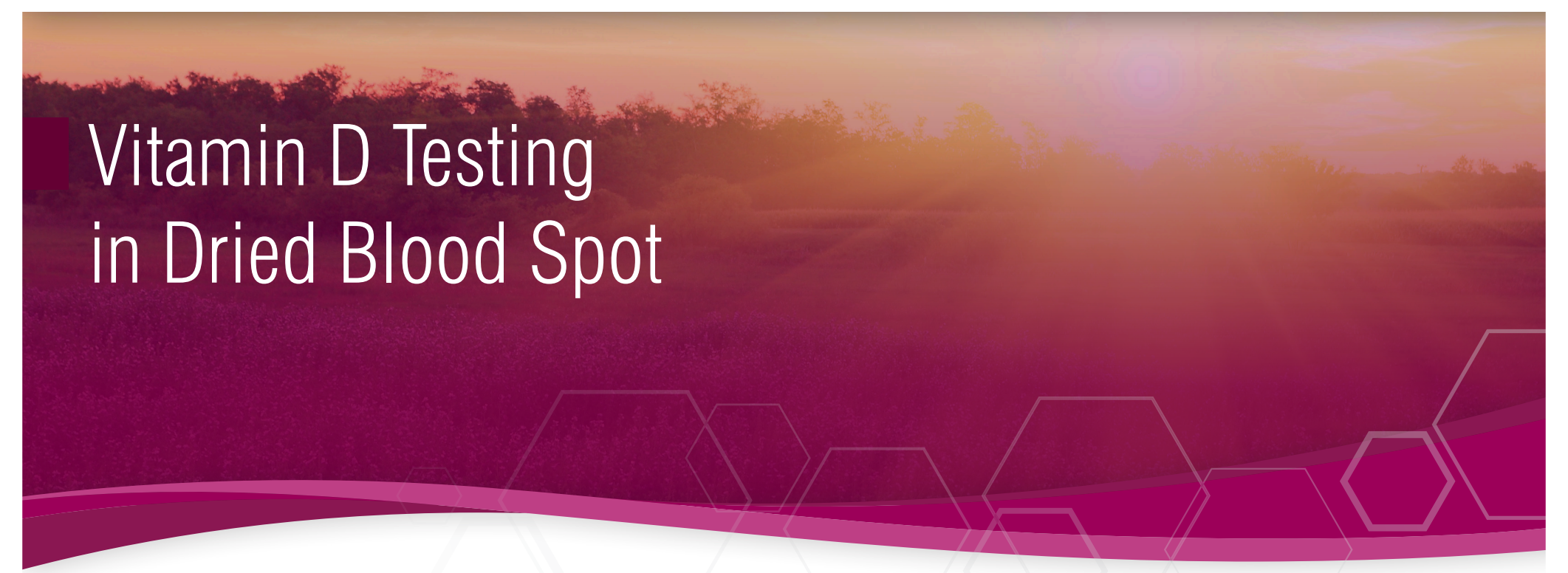

\section{Vitamin D Deficiency}

Long known for its role in the prevention of childhood rickets ${ }^{1}$ and in the intestinal absorption of dietary calcium, vitamin $D$ has now been found to be important in protecting the body from a wide range of diseases. Disorders linked with vitamin D deficiency include stroke, cardiovascular disease, osteoporosis, osteomalacia, several forms of cancer, some autoimmune diseases such as multiple sclerosis, rheumatoid arthritis and type I diabetes, and even type 2 diabetes, depression and schizophrenia ${ }^{2-12}$. A major culprit of vitamin D deficiency is inadequate sun exposure. Vitamin $D$ deficiency is especially problematic for people who spend much of their time indoors, or who live in colder climates.

\section{Who is at Risk?}

\section{- The Elderly}

The vitamin D precursor decreases in the skin with age, therefore elderly people are more prone to deficiency ${ }^{13-16}$. Living in rest homes or becoming home-bound can limit exposure to sunshine. Muscle weakness and osteoporosis associated with vitamin D deficiency make the elderly more susceptible to falling and fracture risk ${ }^{17,18}$. Clinical trials indicate that vitamin D supplementation may decrease the risk of fractures ${ }^{19}$.

\section{- Dark-Skinned People}

Dark skinned people require much longer sunlight exposure to generate adequate circulating vitamin D compared to fair-skinned people.

\section{- People with Limited Sunlight Exposure}

People living at northern latitudes or who have limited sunlight exposure because of their working environment or cultural dress rules may have low vitamin $\mathrm{D}$ levels.

\section{Available Tests}

\section{Vitamin D Panel}

Tests: D2, D3 (blood spot)

Identifies vitamin $D$ deficiency as a potential cause of health problems - levels below $32 \mathrm{ng} / \mathrm{mL}$ are thought to indicate deficiency (research is ongoing to establish definitive recommendations - some experts suggest that levels should be $>50 \mathrm{ng} / \mathrm{mL}$ for optimal health). Also test to monitor a patient's 25-hydroxyvitamin D status following vitamin $D$ supplementation to ensure adequate dosing. 


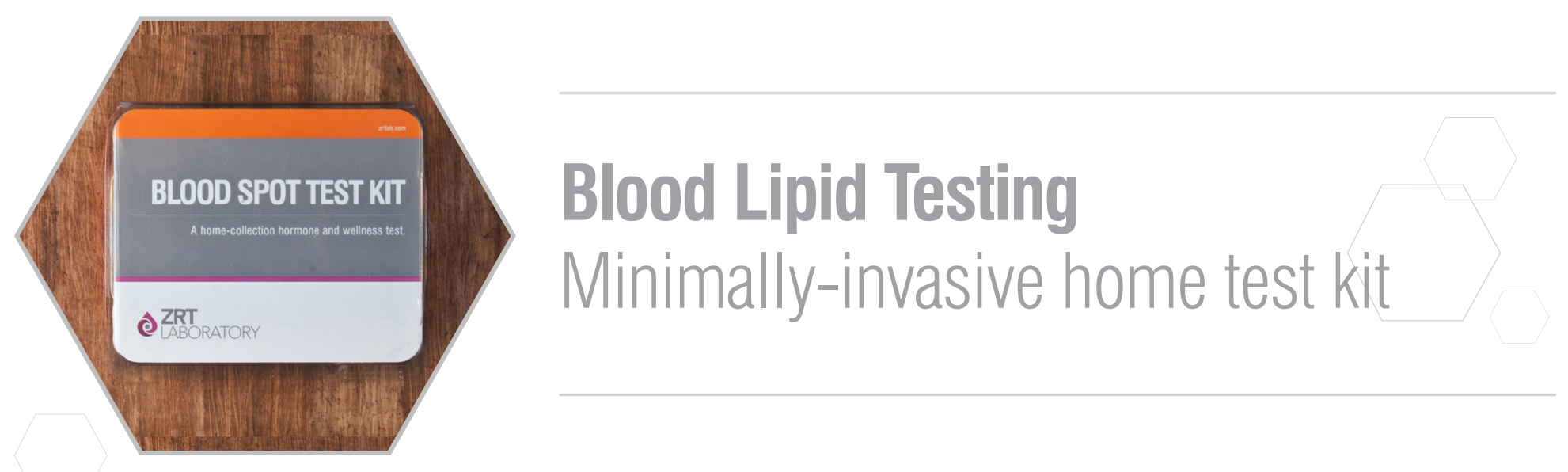

\section{- Musculoskeletal Pain Sufferers}

Patients with symptoms of hypothyroidism ${ }^{20}$, non-specific musculoskeletal pain ${ }^{21}$, chronic low back pain ${ }^{22}$, or fibromyalgia ${ }^{23}$ are frequently found to have low vitamin $D$ levels and show clinical improvement after supplementation. Vitamin D screening is strongly recommended in patients with musculoskeletal pain ${ }^{24}$.

\section{- Overweight or Obese People}

Vitamin D can be locked up in fat stores in obese patients, who have been found to have lower levels of circulating 25-hydroxy vitamin D and are at risk of deficiency ${ }^{25}$.

\section{- Breast-Fed Infants, and Children with Limited Sunlight Exposure}

All children require adequate circulating vitamin $D$ to prevent rickets. Dark-skinned children and those who spend much of the day in indoor daycare centers are at risk of deficiency. Breast-fed children often receive inadequate amounts of vitamin D, particularly when their mothers are deficient. Maternal supplementation ${ }^{26}$ or the use of cod liver oil or other vitamin D supplements in infants and children can avoid the risk of developing type 1 diabetes in childhood ${ }^{27}$.

\section{Why Measure $D$ Levels?}

Vitamin D screening has been recommended as a routine part of the annual physical examination ${ }^{3,5}$. Deficiency may be present even when there are no symptoms, yet it is simple to correct and may solve a number of subclinical health problems and reduce risk for more serious diseases.

\section{D3 vs. D2}

Vitamin D3 (cholecalciferol) is technically a prohormone, and is produced endogenously from the action of ultraviolet light on 7-dehydrocholesterol in the skin. It is also found in cod liver oil and vitamin D supplements. Vitamin D2 (ergocalciferol) is not found in animals, but is manufactured commercially by exposing a component of fungal cell membranes, ergosterol, to ultraviolet light. Vitamin D2 is the predominant form for prescription use in the US, especially in high dose preparations. Both D2 and D3 are hydroxylated in the liver to form their 25-hydroxy metabolites, the major circulating form of the prohormone. The long half-life of 25-hydroxyvitamin D (> 2 weeks) allows for supplementation with large doses every few months in deficient individuals because Vitamin $\mathrm{D}$ is stored by the body in adipose tissue. The 25-hydroxy metabolites are further hydroxylated, primarily in the kidneys, to form 1,25-dihydroxy vitamin D2 and D3. These are the highly active forms of the hormone that bind to specific vitamin $D$ receptors in target tissues and activate specific genes.

Formation of 1,25-dihydroxy vitamin $D$ is tightly regulated by the action of parathyroid hormone. In vitamin $\mathrm{D}$ deficient states, there is excess production of parathyroid hormone (secondary hyperparathyroidism) which can stimulate the kidneys to produce more 1,25-dihydroxyvitamin D, such that levels can appear to be normal or even elevated. For this reason, the 25-hydroxy metabolite, which reflects total body bioavailability of the prohormone, is the commonly accepted measure of vitamin D status.

Since vitamin D2 was effectively used as a supplement to prevent rickets, it has historically been assumed to be equivalent to vitamin D3. However, more recently it has been found that 25-hydroxyvitamin D2 has a lower affinity than D3 
for vitamin D binding protein, which results in a shorter halflife for D2 in the blood stream ${ }^{28,29}$. This makes vitamin D2 less bioactive than D3 therefore D2 must be given in much larger doses. When given daily, D2 is as effective as D3 in maintaining circulating levels of total 25-hydroxyvitamin $D^{30}$. Some vitamin D assays only measure D3 and not D2. In individuals taking D2 this can result in suppression of endogenous D3 and "apparent" vitamin D deficiency. This is important when assessing vitamin $D$ status in people supplementing with D2, which has a greater potential for toxicity than D3 if supplementation is not properly monitored with an accurate assay. The bioequivalence of D2 and D3 is an interesting area for research, particularly since trials showing reduced fractures with vitamin $\mathrm{D}$ supplementation have employed D3 at adequate levels ${ }^{19}$ rather than D2, and many vitamin producers are already switching to D3 in their over-the-counter preparations.

\section{Accuracy of the ZRT Vitamin D Test}

- ZRT uses liquid chromatography/tandem mass spectrometry (LC-MS/MS) ${ }^{31}$ and participates in DEQAS, the Vitamin D Quality Assessment Scheme, which provides control samples to ensure assay accuracy.

- Most published studies, on which currently accepted physicians' recommendations for blood levels are based, have used a radioimmunoassay test from DiaSorin. This test, despite a high correlation with LC-MS/MS for the same samples, usually gives values $20-30 \%$ lower than LC-MS/MS

- Method accuracy is ensured by standardization with the National Institute of Standards and Technology (NIST) Vitamin D standard reference materials.

\section{Clinical Utility}

Blood spot testing for 25-hydroxyvitamin D can help:

- Identify vitamin $\mathrm{D}$ deficiency as a potential cause of health problems - levels below $32 \mathrm{ng} / \mathrm{mL}$ are thought to indicate deficiency ${ }^{32}$ (research is ongoing to establish definitive recommendations - some experts suggest that levels should be $>50 \mathrm{ng} / \mathrm{mL}$ for optimal health ${ }^{33}$ )

- Monitor a patient's 25-hydroxyvitamin D status following vitamin D supplementation to ensure adequate dosing

- Practitioners make appropriate recommendations for vitamin D supplementation, or appropriate lifestyle changes for patients who may benefit from spending time, or exercising, outdoors to increase sunlight exposure

\section{Customer Support}

- ZRT's Hormone Evaluation report includes hormone test results, details of hormone supplements and current symptoms reported by the patient, and ZRT analysis

- The report is returned to the patient or ordering healthcare provider in $5-7$ business days and is also available via secure internet access

- ZRT staff physicians are available for enquiries without appointment, 8:00 a.m. to 5:00 p.m. weekdays 


\section{References}

1. Rajakumar K. Vitamin D, cod-liver oil, sunlight, and rickets: a historical perspective. Pediatrics. 2003;112(2):e132-5.

2. Zittermann $A$. Vitamin $D$ in preventive medicine: are we ignoring the evidence? Br J Nutr. 2003;89(5):552-72.

3. Holick MF. Sunlight and vitamin D for bone health and prevention of autoimmune diseases, cancers, and cardiovascular disease. Am J Clin Nutr. 2004;80(6 Suppl):1678S-88S.

4. Zittermann A, Schleithoff SS, Koerfer R. Putting cardiovascular disease and vitamin $\mathrm{D}$ insufficiency into perspective. $\mathrm{Br} J$ Nutr. 2005;94(4):483-92.

5. Holick MF. The vitamin D epidemic and its health consequences. J Nutr. 2005;135(11):2739S-48S.

6. Holick MF. Vitamin D: important for prevention of osteoporosis, cardiovascular heart disease, type 1 diabetes, autoimmune diseases, and some cancers. South Med J. 2005;98(10):1024-7.

7. Peterlik M, Cross HS. Vitamin D and calcium deficits predispose for multiple chronic diseases. Eur J Clin Invest. 2005;35(5):290-304.

8. Holick MF. High prevalence of vitamin D inadequacy and implications for health. Mayo Clin Proc. 2006;81(3):353-73.

9. Zittermann A. Vitamin $D$ and disease prevention with special reference to cardiovascular disease. Prog Biophys Mol Biol. 2006;92(1):39-48.

10. Poole KE, Loveridge N, Barker PJ, Halsall DJ, Rose C, Reeve J, Warburton EA. Reduced vitamin D in acute stroke. Stroke. 2006;37(1):243-5.

11. Heaney RP. Vitamin D--the iceberg nutrient. I Musculoskelet Neuronal Interact. 2006;6(4):334-5.

12. Holick MF. Vitamin D deficiency. N Engl J Med. 2007;357(3):26681.

13. MacLaughlin J, Holick MF. Aging decreases the capacity of human skin to produce vitamin D3. J Clin Invest. 1985;76(4):1536-8.

14. Need AG, Morris HA, Horowitz M, Nordin C. Effects of skin thickness, age, body fat, and sunlight on serum 25-hydroxyvitamin D. Am J Clin Nutr. 1993;58(6):882-5.

15. Need AG, Horowitz M, Morris HA, Nordin BC. Vitamin D status: effects on parathyroid hormone and 1,25-dihydroxyvitamin $D$ in postmenopausal women. Am J Clin Nutr. 2000;71(6):1577-81.

16. Need AG, O'Loughlin PD, Morris HA, Horowitz M, Nordin BE. The effects of age and other variables on serum parathyroid hormone in postmenopausal women attending an osteoporosis center. J Clin Endocrinol Metab. 2004;89(4):1646-9.

17. Gass M, Dawson-Hughes B. Preventing osteoporosis-related fractures: an overview. Am J Med. 2006;119(4 Suppl 1):S3-S11.

18. Boonen S, Bischoff-Ferrari HA, Cooper C, Lips P, Ljunggren O, Meunier PJ,Reginster JY. Addressing the musculoskeletal components of fracture risk with calcium and vitamin D: a review of the evidence. Calcif Tissue Int. 2006;78(5):257-70.

19. Bischoff-Ferrari HA, Willett WC, Wong JB, Giovannucci E, Dietrich T, Dawson-Hughes B. Fracture prevention with vitamin D supplementation: a meta-analysis of randomized controlled trials. JAMA. 2005;293(18):2257-64.
20. Faiz S, Panunti B, Andrews S. The epidemic of vitamin D deficiency. J La State Med Soc. 2007;159(1):17-20; quiz 20, 55.

21. Plotnikoff GA, Quigley JM. Prevalence of severe hypovitaminosis $D$ in patients with persistent, nonspecific musculoskeletal pain. Mayo Clin Proc. 2003;78(12):1463-70.

22. Al Faraj S, Al Mutairi K. Vitamin D deficiency and chronic low back pain in Saudi Arabia. Spine. 2003;28(2):177-9.

23. Erkal MZ, Wilde J, Bilgin Y, Akinci A, Demir E, Bödeker RH, Mann M, Bretzel RG, Stracke H, Holick MF. High prevalence of vitamin D deficiency, secondary hyperparathyroidism and generalized bone pain in Turkish immigrants in Germany: identification of risk factors. Osteoporos Int. 2006;17(8):1133-40.

24. Lewis PJ. Vitamin D deficiency may have role in chronic low back pain. BMJ. 2005;331(7508):109.

25. Wortsman J, Matsuoka LY, Chen TC, Lu Z, Holick MF. Decreased bioavailability of vitamin D in obesity. Am J Clin Nutr. 2000;72(3):6903.

26. Hollis BW, Wagner CL. Vitamin D requirements during lactation: high-dose maternal supplementation as therapy to prevent hypovitaminosis $\mathrm{D}$ for both the mother and the nursing infant. Am J Clin Nutr. 2004;80(6 Suppl):1752S-8S

27. The EURODIAB Substudy 2 Study Group. Vitamin D supplement in early childhood and risk for Type I (insulin-dependent) diabetes mellitus. Diabetologia. 1999;42(1):51-4.

28. Armas LA, Hollis BW, Heaney RP. Vitamin D2 is much less effective than vitamin D3 in humans. J Clin Endocrinol Metab. 2004;89(11):5387-91.

29. Houghton LA, Vieth R. The case against ergocalciferol (vitamin D2) as a vitamin supplement. Am J Clin Nutr. 2006;84(4):694-7.

30. Holick MF, Biancuzzo RM, Chen TC, Klein EK, Young A, Bibuld D, Reitz R, Salameh W, Ameri A, Tannenbaum AD. Vitamin D2 is as effective as vitamin D3 in maintaining circulating concentrations of 25-hydroxyvitamin D. J Clin Endocrinol Metab. 2008;93(3):677-81.

31. Newman MS, Brandon TR, Groves MN, Gregory WL, Kapur S, Zava DT. A Liquid Chromatography/Tandem Mass Spectrometry Method for Determination of 25-Hydroxy Vitamin D2 and 25-Hydroxy Vitamin D3 in Dried Blood Spots: A Potential Adjunct to Diabetes and Cardiometabolic Risk Screening. J Diabet Sci Technol 2009;3(1):156-162.

32. Hollis BW. Circulating 25-hydroxyvitamin D levels indicative of vitamin D sufficiency: implications for establishing a new effective dietary intake recommendation for vitamin D. J Nutr. 2005;135(2):317-22.

33. Cannell JJ, Vieth R, Willett W, et al. Cod liver oil, vitamin A toxicity, frequent respiratory infections, and the vitamin $D$ deficiency epidemic. Ann Otol Rhinol Laryngol. 2008;117(11):864-70. 